\title{
sciendo
}

10.2478/amset-2021-0013

\section{APPLYING QUALITY IMPROVEMENT APPROACHES FOR THE CONTROL OF CRITICAL MEDICAL PROCESSES IN A HEALTHCARE FACILITY}

\author{
Flaviu Moldovan ${ }^{1}$, Petruta Blaga ${ }^{2}$ \\ ${ }^{I}$ IOSUD Doctoral School, "George Emil Palade" University of Medicine, Pharmacy, Science, and Technology \\ of Targu Mures, Gh. Marinescu str. 38, 540142 Targu Mures, Romania \\ 1'moldovan.flaviu95@yahoo.com \\ 2 "George Emil Palade" University of Medicine, Pharmacy, \\ Science, and Technology of Targu Mures, Gh. Marinescu str. 38, 540142 Targu Mures, Romania \\ 2petruta.blaga@umfst.ro
}

\begin{abstract}
Healthcare facilities face major challenges as patients require for the continuous improvement of the healthcare quality. We have used as research method the study of the scientific literature from the medical databases, and we have identified the categories of processes that ensure the quality in a healthcare facility. It is drawn a detailed map of the basic medical processes which highlights the sequence and interaction of medical processes that take place on requesting patients until they become resolved patients. The particularization of quality improvement methods for the improvement of critical medical processes is presented. By using the Pareto diagram it is analyzed the adverse events associated with healthcare and by employment of Ishikawa diagram it is analyzed the causes of associated infections highlighting the factors that contributed to the increase of confirmed healthcare associated infections, which are assigned to the hospital information system and the hospital monitoring system.
\end{abstract}

Key words: healthcare facility, quality improvement, process map, medical processes, adverse events.

\section{Introduction}

Currently, healthcare facilities face major challenges as patients require for the continuous improvement of the healthcare quality. There are quality improvement programs inspired by the industrial environment, such as "lean manufacturing" [1], which is an excellent tool to meet current healthcare challenges [2]. The adoption of such improvement programs requires a lean evaluation from a much more critical perspective [3] and quantification of sustainability effects $[4,5]$. Terms, methods and practices for assessing the quality of harmonized data may establish a common understanding of strengths and limitations for improving quality [6].

The use of health data is based on contextualized data quality management. Henley-Smith et al [7] demonstrated how the context is applied around data quality characteristics and how a data quality framework is developed to ensure that both quality and context are maintained and addressed throughout the evaluation process of the quality of health data.

Initially developed by manufacturing, quality improvement methods have been slowly adopted by healthcare [8]. Critical control points have been (C) 2021 Published by University Press. This is an open access article under the CC BY-NC-ND license (http://creativecommons.org/licenses/CC-BY-4.0/) 
identified and implemented with the support of cause and effect diagram and Pareto diagram [9].

In this context, the use of quality analysis and evaluation methods is becoming a necessity to ensure patient safety [10].

The objective of this research is to identify the categories of processes in a healthcare facility, to highlight the basic medical processes and to present the particularization of quality improvement methods for the improvement of critical medical processes.

\section{Material and method}

The research methodology consisted in the study of the specialized literature, especially from the PubMed database, in order to identify the current state of knowledge of the researched topic regarding the quality assurance processes in the health care field, the effectiveness of incident reporting and promotion of organizational culture. Results are used for process mapping according to ISO 9001 standard.

Then based on the data collected at the County Emergency Clinical Hospital in Targu Mures it is conducted a case study in order to validate in the health system practice the improvement methods Pareto and Ishikawa diagrams that can contribute to reducing the number of medical incidents and ensure the control of critical medical processes in a healthcare facility.

In the scientific literature there is available research on the effectiveness of incident reporting, which results in: decreased mortality and improved patient survival at 1 year, decreased rate of adverse drug events, improved communication between healthcare professionals about patient safety, improved compliance with work processes and medical care to monitor the patient's condition [11].

Studies have shown that computerized clinical decision support systems for both commercially developed and locally developed systems lead to substantial increases in the identification of their events and adverse drug rates [12]. Effective communication and planning are the most important factors in improving the discharge process and reducing adverse events [13].

A limited number of studies have evaluated the effectiveness of promoting the organizational culture of patient safety on improving health performance. There is some evidence to suggest that organizational culture may be a relevant factor in healthcare performance [14]. For example educational sessions stimulate employee attitude and may lead to improvements in work-related outcomes: attitude, job satisfaction, organizational commitment, and culture. The study by Andres et al [15] concludes that the hospital accreditation process can contribute to changes in staff perceptions of organizational culture, with differentiated views of organizational culture between professional groups.

Team training or tools that support team communication can lead to improved staff perceptions of safety culture, care processes, and better patient safety outcomes such as lower adverse events [16]. Managerial visits to departments have the effect of improving staff perceptions but also the safety culture [16]. The hospitals that have been most successful in changing the hospital culture and reducing the standardized risk mortality rate have presented distinct patterns in member diversity, genuine participation, and conflict management capacity [17].

Overall, all studies consider that there is little evidence to support the link between organizational culture and health performance [18], and articulating the nature of this relationship proves difficult [14]. Current available evidence does not identify effective and generalizable strategies for changing organizational culture [19], although it is estimated that investing in strategies to encourage a highperforming organizational culture can assist hospitals in their efforts to improve clinical outcomes [20].

However, changes that improves the quality of healthcare need to be supported. Hovlid et al [21] have shown that organizational learning contributes to the change of healthcare institutions. Improved understanding of the clinical system, identification and control of quality assurance processes, employment of quality improvement approaches, as well as the change in the mental patterns of employees may influenced the healthcare facility to change its performance.

\section{Results}

\subsection{Quality and medical processes}

Desai and Stamer [22] highlighted the commonly used categories of medical process and outcome measures in a hospital that support the quality improvement initiatives. The analysis of the different quality management systems (QMS) in medical practice reveals significant differences between them [23].

However, a study of the categories of processes encountered in a hospital allows their ordering according to the requirements of the ISO9001 standard [24], in the following categories:

- Management processes: hospital context, establishment of quality policy and quality objectives, risk and opportunity management, patient orientation, management analysis;

- Basic medical processes: development of medical services, on-call medical services, specialized clinical medical services, operating rooms, specialized outpatient medical services, medical research, establishing the identity of hospitalized patients without identity documents, occupational medicine;

- Medical support processes: laboratory medical tests, imaging, home care, sterilization, transfusions, nuclear medicine, endoscopy, pharmacy, control and prevention of nosocomial infections; 


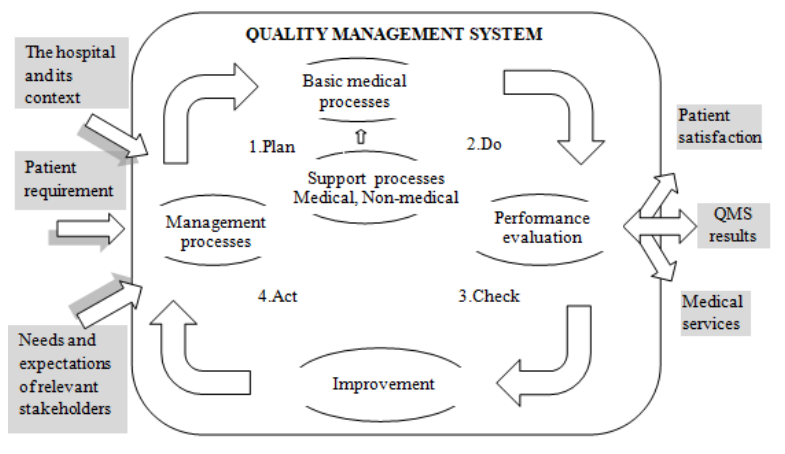

Figure 1. Process map

- Non-medical support processes: control of infrastructure and environment for medical services, internal and external communication, competence and awareness, administrative, procurement, public procurement, financial - accounting, provision of food for inpatients, cleaning, linen handling, control of documented information, measurement and monitoring resource control, transport and storage, infrastructure maintenance;

- Performance appraisal and improvement processes: monitoring and measurement services, patient satisfaction measurement, internal audit, noncompliance treatment and corrective actions, statistics - medical informatics, improvement.

The interaction and succession of the identified processes of the quality management system can be represented in the process map (Fig. 1). In this context, a map of the basic medical processes can be detailed. It highlights the succession and interaction of the medical processes that take place on the requesting patients until they become solved patients (Fig. 2).

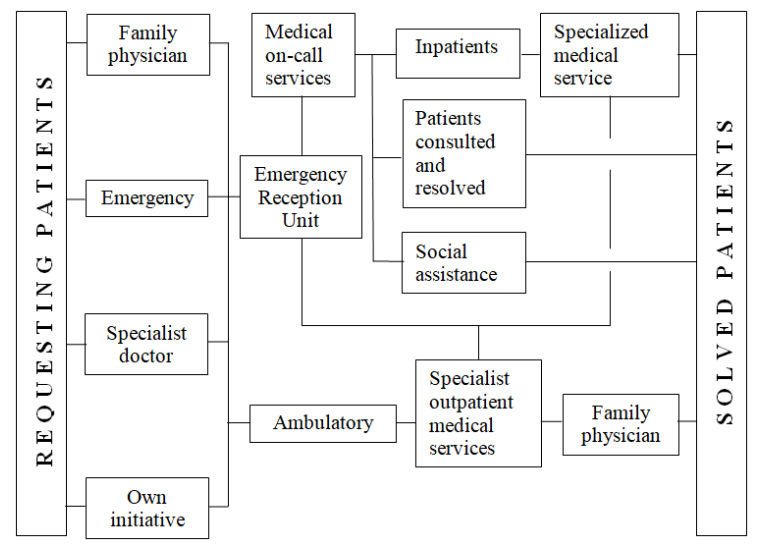

Figure 2. Map of basic medical processes

In hospitals, basic medical processes are affected by adverse events / sentinel / "near-miss" [25]. These events can cause harm to the patient that can lead to increased suffering, resulting in increased hospital stay or even death (in which case it is a sentinel event). Adverse events are monitored and reported in order to identify the causes of occurrence and establish preventive measures [26].

\subsection{Analysis of adverse events associated with healthcare}

The Pareto diagram allows highlighting the most important elements of adverse events associated with health care (AEAHC), on which action must be taken as a matter of priority. In this case we have used the Pareto diagram for the analysis of data collected at the County Emergency Clinical Hospital in Targu Mures (CECHTM) [27], when the types of events and their causes are well known, in order to identify events to be acted upon priority.

The types of adverse events associated with health care were evaluated in the chosen time interval, namely the year 2020, and these were classified according to the causes defined by the National Authority for Quality Management in Health.

Table 1 records on the lines the data collected about adverse events, which are ordered according to their frequency of occurrence. In the first line of the table it is placed the adverse events with the highest frequency of occurrence. Column 3 of the table contains the cumulative number of adverse events.

Table 1. Number of Adverse Events Associated with Health Care, CECHTM, 2020

\begin{tabular}{|c|c|c|c|c|}
\hline $\begin{array}{l}\dot{\Xi} \\
\dot{0} \\
\dot{Z}\end{array}$ & $\begin{array}{c}\text { Associated Adverse } \\
\text { Events } \\
\text { Health Care (AEAHC) }\end{array}$ & 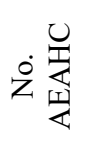 & 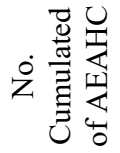 & $\begin{array}{c}\text { Specific } \\
\text { weight } \\
{[\%]}\end{array}$ \\
\hline 0 & 1 & 2 & 3 & 4 \\
\hline 1 & $\begin{array}{l}\text { Infections associated } \\
\text { with confirmed } \\
\text { healthcare }\end{array}$ & 368 & 368 & 90,41 \\
\hline 2 & Falling & 20 & 388 & 95,33 \\
\hline 3 & Hospital leave & 11 & 399 & 98,03 \\
\hline 4 & $\begin{array}{l}\text { Infections associated } \\
\text { with denied health care }\end{array}$ & 5 & 404 & 99,26 \\
\hline 5 & Damage to a branula & 1 & 405 & 99,50 \\
\hline 6 & Aggressive behavior & 1 & 406 & 99,75 \\
\hline 7 & $\begin{array}{l}\text { Post-transfusion adverse } \\
\text { reaction }\end{array}$ & 1 & 407 & 100 \\
\hline & Total AEAHC & & 407 & \\
\hline
\end{tabular}

In figure 3 we have represented the Pareto diagram regarding Adverse Events Associated to Health Care registered at the Targu Mures County Emergency

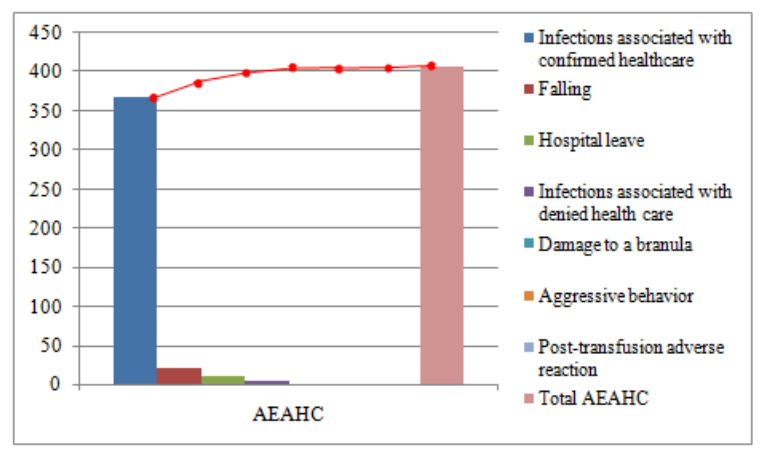

Figure 3. Pareto Diagram on Adverse Events Associated with Health Care at CECHTM in 2020 
Clinical Hospital [27] in 2020. Adverse events are represented on the diagram columns in descending order of reported number of events.

The right corner of each column signifies the cumulative number of events, which are joined by dots and the sum curve of event types is obtained by summing the frequency of all events. From the end of the sum curve a parallel to the ordinate descends. The endpoint represents the $100 \%$ percent of events, according to which the scale for the cumulative percentage is established.

After plotting the sum curve of the types of events, all the categories of events that together give a frequency of $70 \%$, are considered as rank I events. The event categories between $90-100 \%$ are considered as rank III. The remaining types of events are classified as belonging to category II. This classification can also be highlighted with the support of information in column 4 of table 1, which indicates the specific gravity.

The analysis of the diagram shows that infections associated with confirmed healthcare are considered rank I events, which have a frequency of $90.41 \%$, requiring the factors study that contribute to their growth.

\subsection{Analysis of the causes of associated infections}

In the present study, we have identified the factors that contributed to the increase in the rate of infections associated with confirmed healthcare. We conducted interviews and discussions with CECHTM staff, from which we identified a wide variety of causes that were organized in the Ishikawa diagram in Figure 4.

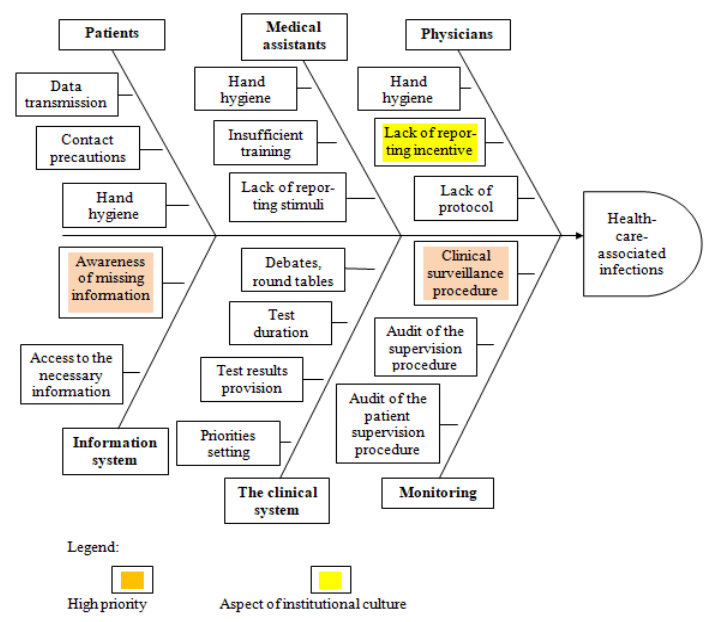

Figure 4. The Ishikawa diagram for the study of the increase in the rate of infections associated with confirmed healthcare

The first-order causes are on the one hand the people who participate in the medical act: patients, nurses and physicians, and on the other hand the hospital subsystems: the information system, the clinical system and the way in which the monitoring of hospital processes is ensured.
Among the second-order causes, the elements that require the most attention and the highest priority approach are represented in color: awareness of the missing information and the clinical surveillance procedure. We also identified an issue related to institutional culture, the lack of reporting incentives.

\section{Conclusion}

By using the Pareto diagram, the quantitative analysis of adverse events associated with health care shows that adverse events are never evenly distributed across the quality characteristics of healthcare. The non-uniformity of the distribution of events is such that a small percentage, respectively a few adverse events of vital importance, has a significant share in the total losses related to the quality of healthcare.

Through this, the analysis provides information about the events that need to be eliminated in order to obtain the highest gains. Therefore, Pareto analysis is appropriate for improving the quality of care in which the initial condition is compared with the condition after the application of improvement measures. In the present case, the events associated with the confirmed healthcare, which have a share of $90.41 \%$ and on which action must be taken as a matter of priority, through reduction and elimination measures, are considered rank I events. Following the application of these measures, a new Pareto diagram can highlight the effect of the proposed measures and the progress made.

The Ishikawa diagram highlighted the factors that contributed to the increase in the rate of confirmed healthcare associated infections, which are attributed to the hospital information system, and requires reanalyzing the types of medical and administrative information and supplementing the information system with information that is not transmitted, as well as the hospital monitoring system, requiring a review and improvement of the clinical surveillance procedure as soon as possible.

These findings are in line with the main studies in the scientific literature, which highlight the importance of communicating information, with direct effects on creating a culture of medical quality in ensuring basic medical processes.

\section{References}

[1] Moldovan, F. (2017), Aplicarea principiilor Lean în asistenta medicala [Applying the principles of Lean in healthcare], Review of Management and Economic Engineering, vol. 16(3), pp. 69-570.

[2] Van Den Heuvel, J., Does, R.J.M.M., and De Koning H. (2006), Lean Six Sigma in a Hospital, International Journal of Six Sigma and Competitive Advantage, vol. 2(4), pp 377-388.

[3] Brandao de Souza, L. (2009), Trends and Approaches in Lean Healthcare, Leadership in Health Service, vol. 22(2), pp 121-139.

[4] Kruk M.E., Gage, A.D., Arsenault, C., et al. (2018), High-quality health systems in the Sustainable 
Development Goals era: time for a revolution, The Lancet Global Health, vol. 6(11), pp. 1-57.

[5] Moldovan, F. (2020), Framework Specifications for Evaluation of Quality Improvement and Sustainable Development in Healthcare Facilities, Proceedings, vol. 63(1), 2.

[6] Kahn, M.G., Callahan, T.J., Barnard, J., et al. (2016), Harmonized Data Quality Assessment Terminology and Framework for the Secondary Use of Electronic Health Record Data. EGEMS (Wash DC)., vol. 4(1), 1244.

[7] Henley-Smith, S., Boyle, D., and Gray, K. (2019), Improving a Secondary Use Health Data Warehouse: Proposing a Multi-Level Data Quality Framework, EGEMS (Wash DC), vol. 7(1), 38.

[8] Picarillo, A.P. (2018), Introduction to quality improvement tools for the clinician. J Perinatol., vol. 38(7), pp. 929-935.

[9] Varzakas, T.H., and Arvanitoyannis, I.S. (2007), Application of Failure Mode and Effect Analysis (FMEA), cause and effect analysis, and Pareto diagram in conjunction with HACCP to a corn curl manufacturing plant. Crit Rev Food Sci Nutr., vol. 47(4), pp. 363-387.

[10] Moldovan, F. (2018), New Approaches and Trends in Health Care, Procedia Manufacturing, vol. 22, pp. 947-951.

[11] Percarpio, K.B., Watts B.V., and Weeks W.B. (2008), The effectiveness of root cause analysis: what does the literature tell us?, Jt Comm J Qual Patient Saf., vol. 34(7), pp. 391-398.

[12] Kaushal, R., Shojania, K.G., and Bates, D.W. (2003), Effects of computerized physician order entry and clinical decision support systems on medication safety: a systematic review, In: Database of Abstracts of Reviews of Effects (DARE). [Online]. Available: https://www.ncbi.nlm.nih.gov/books/NBK70091/

[13] Braet, A., Weltens, C., and Sermeus, W. (2016), Effectiveness of discharge interventions from hospital to home on hospital readmissions: a systematic review, JBI Database System Rev Implement Rep., vol. 14(2), pp. 106-73.

[14] Scott, T., Mannion, R., Marshall, M., and Davies, H. (2003), Does organisational culture influence health care performance? A review of the evidence, J Health Serv Res Policy., vol. 8(2), pp. 105-17.

[15] Andres, E.B., Song, W., Schooling, C.M., and Johnston, J.M. (2019), The influence of hospital accreditation: a longitudinal assessment of organisational culture, BMC Health Serv Res., vol. 19, 467.

[16] Weaver, S.J., Lubomski, L.H., Wilson, R.F., Pfoh E.R., Martinez, K.A., and Dy S.M. (2013), Promoting a culture of safety as a patient safety strategy: a systematic review, In: Database of Abstracts of Reviews of Effects (DARE). [Online].
Available:https://www.ncbi.nlm.nih.gov/books/N BK126977/

[17] Bradley, E.H., Brewster, A.L., McNatt, Z., Linnander E.L., Cherlin, E., Fosburgh, H., Ting, H.H., and Curry, L.A. (2018), How guiding coalitions promote positive culture change in hospitals: a longitudinal mixed methods interventional study, BMJ Quality \& Safety, vol. 27, pp.218-225.

[18] Morello, R.T., Lowthian, J.A., Barker, A.L., McGinnes, R., Dunt, D. and Brand, C. (2013), Strategies for improving patient safety culture in hospitals: a systematic review, BMJ Qual Saf., vol. 22(1), pp.11-8.

[19] Parmelli, E., Flodgren, G., Beyer, F., Baillie N., Schaafsma M.E., and Eccles M.P. (2011), The effectiveness of strategies to change organisational culture to improve healthcare performance: a systematic review, Implementation Sci., vol. 6, 33.

[20] Curry, L.A., Brault, M.A., Linnander, E.L., McNatt, Z., Brewster, A.L., Cherlin, E., Flieger, S.P., Ting, H.H., and Bradley, E.H. (2018), Influencing organisational culture to improve hospital performance in care of patients with acute myocardial infarction: a mixed-methods intervention study, BMJ Quality \& Safety, vol. 27, pp. 207-217.

[21] Hovlid, E., Bukve, O., Haug, K., Aslaksen, A.B., and von Plessen, C. (2012), Sustainability of Healthcare Improvement: What Can We Learn from Learning Theory? BMC Health Services Research, vol. 12(235), pp 1-13.

[22] Desai, A.D., and Starmer, A.J. (2019), Process Metrics and Outcomes to Inform Quality Improvement in Pediatric Hospital Medicine, Pediatr Clin North Am., vol. 66(4), pp. 725-737.

[23] Gumpert, M. and Reese, J.P. (2019), Analyse verschiedener Qualitätsmanagementsysteme in Arztpraxen für Sachsen [Analysis of Different Quality Management Systems in Medical Practices in Saxony], Gesundheitswesen, vol. 81(12), pp. 1037-1047.

[24] ISO 9001:2015. Quality management systems Requirements. [Online]. Available: https://www.iso.org/standard/62085.html

[25] Pettker, C.M. (2017), Systematic approaches to adverse events in obstetrics, Part I: Event identification and classification. Semin Perinatol., vol. 41(3), pp. 151-155.

[26] Singal, M., Zafar, A., Tbakhi, B., Jadhav, N., Alweis, R., and Bhavsar, H. (2018), Assessment of knowledge and attitudes towards safety events reporting among residents in a community health system. J Community Hosp Intern Med Perspect., vol. 8(5), pp. 253-259.

[27] County Emergency Clinical Hospital of TarguMures. [Online]. Available: https://www.spitalmures.ro/desprespital/prezentare-generala/ 